\title{
ARBEKACIN - A RAY OF HOPE TO FIGHT AGAINST MDR AND XDR GRAM-NEGATIVE BACTERIA IN A SCIENTIFIC AND COST-EFFECTIVE WAY IN INDIAN SCENARIO
}

\author{
SOMA SARKAR ${ }^{1}$, DIPANKAR SARKAR ${ }^{2 *}$, ANJUM NAMHATA $^{1}$, MANIDEEPA SENGUPTA ${ }^{3}$
}

${ }^{1}$ Department of Microbiology, NRS Medical College, Kolkata, West Bengal, India. ${ }^{2}$ Internist and Intensivist, ICU Incharge, Columbia Asia Hospital, Salt-lake, Kolkata, West Bengal, India. ${ }^{3}$ Department of Microbiology, Medical College, Kolkata, West Bengal, India.

\author{
Email: drdipsoma@gmail.com
}

Received: 08 February 2020, Revised and Accepted: 18 March 2020

ABSTRACT

Objective: The objective of the study was to see the in vitro activity of arbekacin, a novel aminoglycoside, against multidrug-resistant (MDR) and extensively drug-resistant (XDR) Gram-negative bacilli (GNB) so that it can become a good alternative as empirical treatment for severe sepsis.

Methods: Identification and antibiotic sensitivity testing of the GNB isolated from the clinical samples were done using the VITEK-II system in a tertiary care hospital, Kolkata. MDR and XDR strains were selected by their definitions and molecular characterization was done by multiplex polymerase chain reaction. The minimum inhibitory concentration (MIC) value of arbekacin was detected by the E-test strip and compared with other aminoglycosides.

Results: A total of 140 drug-resistant strains including ESBL- and carbapenemase-producing GNB were selected for the study. Arbekacin showed reduced values of MIC $_{50}$ and MIC $_{90}$ compared to other aminoglycosides for most of the drug-resistant GNB.

Conclusion: Hence, in this drug-resistant era, arbekacin with the advantage of a single daily dose can be used as an empirical choice in severe sepsis as monotherapy or in combination with other antibiotics such as colistin or polymyxin to fight against MDR and XDR bugs.

Keywords: Aminoglycoside, Arbekacin, Gram-negative bacilli, Multidrug resistant, Extensively drug resistant.

(C) 2020 The Authors. Published by Innovare Academic Sciences Pvt Ltd. This is an open access article under the CC BY license (http://creativecommons. org/licenses/by/4. 0/) DOI: http://dx.doi.org/10.22159/ajpcr.2020.v13i5.37069

\section{INTRODUCTION}

Antimicrobial resistance poses a major threat to public health and makes therapeutic decisions more challenging. India carries one of the largest burdens of drug-resistant pathogens worldwide [1]. Emergence of their multidrug-resistant (MDR) and extensively drugresistant (XDR) Gram-negative pathogens adds on to the complexity of infection, rendering them difficult to treat [2]. As there are not enough new antibiotics in the pipeline to meet the challenge of antibiotic resistance, the pharmaceutical industry suggests drug repurposing, to find new uses of old drugs [3]. The drug arbekacin had been licensed in Japan for use under the trade name "Habekacin" for the treatment of MRSA caused sepsis and pneumonia since 1990 [4,5]. Arbekacin is an aminoglycoside dideoxykanamycin B (dibekacin) belonging to the kanamycin family [6]. Arbekacin has the structure of 1-N-[(S)4-amino-2-hydroxybutyryl (AHB)]-DKB. Due to the introduction of $\mathrm{AHB}$ to $1-\mathrm{N}$ position of $\mathrm{DKB}$, the resulting arbekacin exhibited activity against drug-resistant bacteria [7]. Arbekacin is stable to the aminoglycoside-inactivating enzymes such as aminoglycoside phosphotransferase (APH), aminoglycoside adenylyltransferase (AAD), and aminoglycoside acetyltransferase, whereas gentamicin, amikacin, tobramycin, and kanamycin were completely inactivated by APH (2") [8]. Arbekacin is a good bactericidal drug against MRSA, enterococci and shows concentration-dependent and long-lasting postantibiotic effects [9-12], but its activity against MDR and XDR Gramnegative bacilli (GNB) is not evaluated. Hence, the objective of the study was to see the in vitro activity of arbekacin against MDR and XDR GNB isolated from different clinical samples.

\section{METHODS}

Identification of the isolates from clinical samples

The study was conducted in the Department of Microbiology, NRS Medical College, Kolkata, from May 2019 to August 2019. Isolation of
GNB was done from different samples (urine, respiratory samples, pus, and blood) by standard microbiological procedure and identification up to the species level was done using VITEK-GN cards (bioMérieux India Private Limited).

\section{Antimicrobial susceptibility testing}

Antibiotic sensitivity testing was performed in the VITEK-2 system using AST-GN280 and AST-GN281 susceptibility cards and interpreted according to the Clinical and Laboratory Standards Institute recommendations. P. aeruginosa ATCC 27853 and Escherichia coli ATCC 25922 were used as quality control strains.

\section{Inclusion and exclusion criteria}

Selection for MDR and XDR strains from the isolated GNB was based on the definition of MDR (acquired non-susceptibility to at least one agent in three or more antimicrobial categories) and XDR [non-susceptibility to at least one agent in all but two or fewer antimicrobial categories (i.e., bacterial isolates remain susceptible to only one or two antimicrobial categories)]. Isolated GNB strains sensitive or resistant to one/two antimicrobial categories were excluded from the study.

Molecular characterization of MDR and XDR Gram-negative strains The presence of carbapenemase-encoding genes from the selected MDR and XDR GNB was determined by multiplex PCR using primers (ReadyMade $^{\mathrm{TM}}$ Primers, Integrated DNA Technologies) (Table 1) targeting blaVIM, blaIMP, blaKPC, blaOXA-48, and blaNDM (Fig. 1).

ESBL production was determined by placing ceftazidime (CAZ $30 \mu \mathrm{g}$ ) disks with or without clavulanic acid (CA $10 \mu \mathrm{g}$ ) on MHA plate. After overnight incubation, if there was an augmentation of $\geq 5 \mathrm{~mm}$ in the inhibitory zone diameter of CAZ-CA in comparison to CAZ alone, ESBL by that strain was phenotypically confirmed. 
The minimum inhibitory concentration (MIC) of arbekacin against MDR and XDR Gram-negative bacterial isolates

To calculate the MIC of arbekacin by E-test strip, a lawn culture is made by the organism to be tested, over a Mueller-Hinton agar plate and the

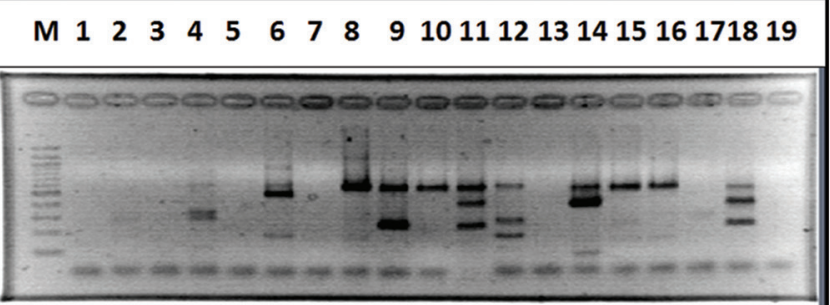

Fig. 1: Multiplex PCR for the detection of carbapenemaseproducing genes. $M, 100$ bp DNA ladder lane: 6, 8, 10, 15, 16 NDM positive (603 bp) Lane: 9, 12 NDM+ OXA-48 (265 bp) positive bacteria. Lane:11, 18 positive control. Lane: 19 negative control

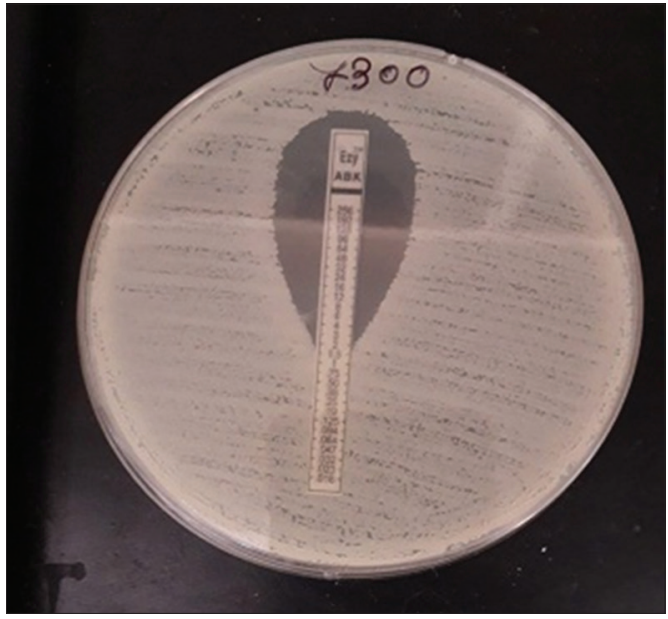

Fig. 2: Arbekacin E-test arbekacin E-test strip was applied onto it. After overnight incubation, the reading of MIC was taken as the value at the point where ellipse intersects the scale (Fig. 2).

\section{RESULTS}

Identification of the isolates from clinical samples

A total of 1276 GNB were isolated during the study period. Of them, 526 were E. coli, 314 were Klebsiella pneumoniae, 24 were Enterobacter cloacae, 48 were Proteus spp., 190 were P. aeruginosa, and 174 were Acinetobacter spp.

Among them, 140 representatives drug-resistant GNB were selected according to their antibiotic resistance pattern (Table 2). Of them, 30 were E. coli, 55 were K. pneumoniae, 12 were E. cloacae, 3 were Proteus spp., 24 were P. aeruginosa, and 16 were Acinetobacter spp. Arbekacin breakpoints were used according to Lee et al., 2007 [13], as susceptible $\leq 4 \mu \mathrm{g} / \mathrm{mL}$; intermediate $8 \mu \mathrm{g} / \mathrm{mL}$; and resistant $\geq 16 \mu \mathrm{g} / \mathrm{mL}$.

\section{Antimicrobial susceptibility testing}

Arbekacin showed $50 \%$ resistance to E. coli, 54.55\% resistance to Klebsiella spp., $33.34 \%$ resistance to E. cloacae, $66.67 \%$ resistance to Proteus spp., 20\% resistance to Pseudomonas spp., and 50\% resistance to Acinetobacter spp. (Table 3).

According to the resistance pattern of the selected Gram-negative isolates for the study, 50 strains are MDR and 90 strains are XDR (Table 4).

Among 50 MDR Gram-negative strains, 34 (68\%) are sensitive and $16(32 \%)$ are resistant to arbekacin, whereas among 90 XDR Gramnegative strains, $43(47.78 \%)$ are sensitive to arbekacin and 47 (52.23\%) are resistant.

The MIC of arbekacin in MDR and XDR Gram-negative strains $\mathrm{MIC}_{50}$ and $\mathrm{MIC}_{90}$ of arbekacin for E. coli were 1.5 and 16, for K. pneumoniae 16 and 64, for E. cloacae 0.75 and 8, for Proteus spp. 24 and $>128$, for Pseudomonas aeruginosa 1.5 and 8, and Acinetobacter spp. 4 and 64, respectively (Table 5).

Table 1: Primer sequences and amplicon sizes

\begin{tabular}{|c|c|c|c|}
\hline PCR name & Targeted gene & Primer sequence ( $5^{\prime}$ to $\left.3^{\prime}\right)$ & Amplicon size \\
\hline \multirow[t]{7}{*}{ CARBA } & blaNDM & $\begin{array}{l}\text { Forward ACT TGG CCT TGC TGT CCT T } \\
\text { Reverse CAT TAG CCG CTG CAT TGA T }\end{array}$ & $603 \mathrm{bp}$ \\
\hline & blaVIM & Forward TGT CCG TGA TGG TGA TGA G T & 437 bp \\
\hline & & Reverse ATT CAG CCA GAT CGG CAT C & \\
\hline & blaIMP & Forward ACA YGG YTT RGT DGT KCT TG & $387 \mathrm{bp}$ \\
\hline & & Reverse GGT TTA AYA AAR CAA CCA CC & \\
\hline & bla0XA-48 & Forward ATG CGT GTA TTA GCC TTA TCG & $265 \mathrm{bp}$ \\
\hline & & Reverse CAT CCT TAA CCA CGC CCA AAT C & \\
\hline \multirow[t]{2}{*}{ OXA CARBA } & blaOXA-23group & Forward CCC CGA GTC AGA TTG TTC AAG G & $330 \mathrm{bp}$ \\
\hline & & Reverse TAC GTC GCG CAA GTT CCT GA & \\
\hline
\end{tabular}

PCR: Polymerase chain reaction, CARBA: Carbapenemase, NDM: New Delhi metallo- $\beta$-lactamase, VIM: Verona integron-encoded metallo- $\beta$-lactamase, IMP: Imipenemase, KPC: Klebsiella pneumoniae carbapenemase, OXA: Oxacillinase

Table 2: Isolation and identification of selected MDR and XDR GNB from different sources

\begin{tabular}{llllll}
\hline & Urine & Wound swab & Blood & Sputum and endotracheal aspirates & Total \\
\hline Escherichia coli & 10 & - & 20 & - & 30 \\
Klebsiella pneumoniae & 30 & 5 & 15 & 5 & 55 \\
Enterobacter cloacae & 10 & 2 & - & - & 12 \\
Proteus spp. & 1 & 2 & 5 & - & 3 \\
Pseudomonas aeruginosa & 5 & 9 & - & 11 & 24 \\
Acinetobacter baumannii & - & 5 & 40 & 21 & 16 \\
Total & 56 & 23 & &
\end{tabular}




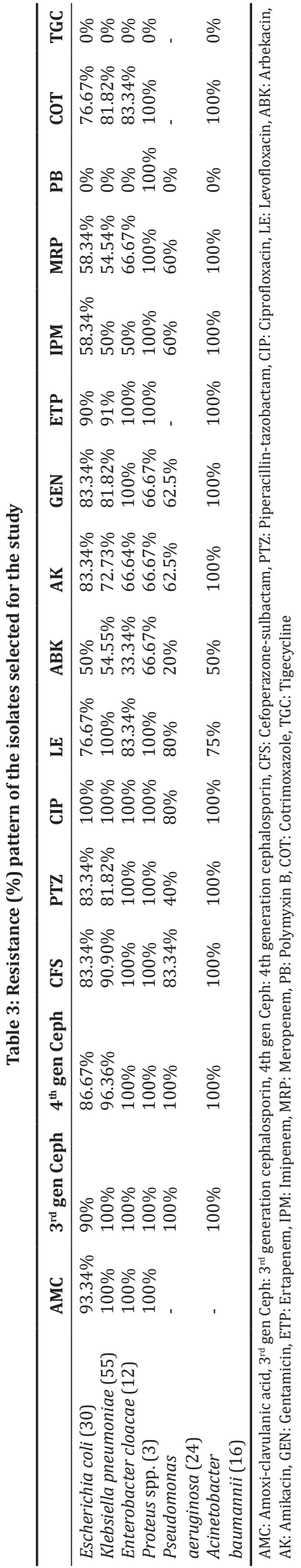

\section{DISCUSSION}

The increased prevalence of MDR and XDR GNB along with their rapid spread is a matter of concern in modern medicine. In our study period over 4 months, we have selected 140 drug-resistant strains including ESBL and carbapenemase-producing GNB which were representative of MDR and XDR strains among the total of 1276 isolates. We compared the resistance pattern of arbekacin with other aminoglycosides (amikacin and gentamicin) and other potent antimicrobial drugs with these selected isolates. Arbekacin showed a better sensitivity profile than penicillin, cephalosporin, other aminoglycosides, fluoroquinolones, $\beta$-lactam- $\beta$-lactamase inhibitors, and carbapenems. Polymyxin B and tigecycline showed better sensitivity than arbekacin. In Proteus spp., the sensitivity of arbekacin was comparable with other aminoglycosides.

This study showed that $\mathrm{MIC}_{50}$ of arbekacin was 10.67 times lower than that of amikacin and gentamicin for E. coli, 4 times lower than that of amikacin, and 2 times lower than gentamicin in Klebsiella spp., 1.33 times lower than that of amikacin, and 2.67 times lower than that of gentamicin for Enterobacter spp., 1.33 times lower than that of amikacin but $\mathrm{MIC}_{50}$ of gentamicin was 3 times lower than that of arbekacin in Proteus spp. In the case of Pseudomonas spp., $\mathrm{MIC}_{50}$ of arbekacin is 5.33 times lower than that of amikacin and gentamicin and in Acinetobacter spp., arbekacin $\mathrm{MIC}_{50}$ was 8 times lower than amikacin and 4 times lower than gentamicin. However, the value of MIC of arbekacin was high in Klebsiella $\left(\mathrm{MIC}_{50}=16, \mathrm{MIC}_{90} \geq 64\right.$ ) and Proteus $\left(\mathrm{MIC}_{50}=24, \mathrm{MIC}_{90} \geq 128\right.$ ) (Table 5). In these cases, we might consider the combination therapy with polymyxins/tigecycline or carbapenem (if carbapenem was sensitive) [14]. Antibiotic combination therapy study groups suggested the effective combination of arbekacin plus aztreonam against MDR Pseudomonas [15].

Hence, the MIC value of arbekacin is lower than other aminoglycosides in drug-resistant GNB which was similar to the study by Watanabe et al., 2012, Kazuno et al., 1986 [16,17]. Arbekacin is a unique aminoglycoside because of its ability to concentrate in pulmonary epithelial lining fluid [18], unlike other aminoglycosides which will help us to use it against pneumonia and other respiratory diseases. Again, simultaneous Gram-positive and Gram-negative coverage with a single daily dose are the advantage of arbekacin and it can be used as an empirical choice in severe sepsis. To interpret its in vitro activity, the sensitivity of other aminoglycosides (amikacin and gentamicin) can guide arbekacin sensitivity for treating physicians in a resource-limited setting. Although the sensitivity of tigecycline is better than arbekacin, the favorable pharmacodynamics of arbekacin make the drug superior to tigecycline.

A higher dose of arbekacin can improve clinical efficacy. In a study by Matsumoto et al., 2014 [8], it was shown that in patients with normal renal function, the target peak concentration (Cpeak) was not achieved with once-daily administration of 150-200 $\mathrm{mg}$ and a higher dosing regimen is required to improve clinical efficacy, and the Japanese Society of Chemotherapy and the Japanese Society of Therapeutic Drug Monitoring Experts recommend 5.5-6.0 mg/kg body weight to reach the target concentration [19]. The pharmacokinetics in healthy volunteers with normal renal function did not change on 400 and $600 \mathrm{mg}$ single dose and the total clearance does not decrease at a high dose [8].

\section{Table 4: Number of MDR and XDR strains selected for the study}

\begin{tabular}{lll}
\hline & MDR strains & XDR strains \\
\hline Escherichia coli & 10 & 20 \\
Klebsiella pneumoniae & 20 & 35 \\
Enterobacter cloacae & 9 & 3 \\
Proteus spp. & 0 & 3 \\
Pseudomonas aeruginosa & 11 & 13 \\
Acinetobacter baumannii & 0 & 16 \\
\hline
\end{tabular}

MDR: Multidrug resistant, XDR: Extensively drug resistant 
Table 5: Comparison of MIC of arbekacin with gentamicin and amikacin against selected drug resistant Gram-negative isolates

\begin{tabular}{|c|c|c|c|c|c|c|c|c|}
\hline \multirow[t]{2}{*}{ Isolates } & \multirow[t]{2}{*}{$\begin{array}{l}\text { Molecular characterization of the } \\
\text { isolates }\end{array}$} & \multicolumn{2}{|c|}{$\begin{array}{l}\text { Arbekacin MIC } \\
(\mu \mathrm{g} / \mathrm{ml})\end{array}$} & \multicolumn{2}{|c|}{$\begin{array}{l}\text { Amikacin MIC } \\
(\mu \mathrm{g} / \mathrm{ml})\end{array}$} & \multicolumn{2}{|c|}{$\begin{array}{l}\text { Gentamicin MIC } \\
(\mu \mathrm{g} / \mathrm{ml})\end{array}$} & \multirow[t]{2}{*}{$\begin{array}{l}\text { Range of MIC in } \\
\text { arbekacin }(\mu \mathrm{g} / \mathrm{ml})\end{array}$} \\
\hline & & MIC $_{50}$ & MIC $_{90}$ & MIC $_{50}$ & MIC $_{90}$ & MIC $_{50}$ & MIC $_{90}$ & \\
\hline $\begin{array}{l}\text { Klebsiella pneumoniae } \\
(\mathrm{n}=55)\end{array}$ & $\begin{array}{l}8 \text { are NDM+OXA4 } 8 \text { producing, } 19 \\
\text { are NDM producing and } 38 \text { are ESBL } \\
\text { producing }\end{array}$ & 16 & $>64$ & 64 & $>128$ & 32 & $>128$ & $1->128$ \\
\hline $\begin{array}{l}\text { Enterobacter cloacae } \\
(\mathrm{n}=12)\end{array}$ & 6 are ESBL producing & 0.75 & 8 & 1 & 32 & 2 & 64 & $0.25-1$ \\
\hline Proteus spp. $(\mathrm{n}=3)$ & 3 are ESBL producing & 24 & $>128$ & 32 & $>128$ & 8 & $>128$ & $8->128$ \\
\hline $\begin{array}{l}\text { Pseudomonas } \\
\text { aeruginosa }(\mathrm{n}=24)\end{array}$ & 9 are ESBL producing & 1.5 & 8 & 8 & 64 & 8 & $>64$ & $0.19-16$ \\
\hline $\begin{array}{l}\text { Acinetobacter } \\
\text { baumannii }(\mathrm{n}=16)\end{array}$ & $\begin{array}{l}7 \text { are ESBL producing and } 9 \text { are } \\
\text { NDM+OXA23 producing }\end{array}$ & 4 & 64 & 32 & $>128$ & 16 & $>128$ & $0.25->64$ \\
\hline
\end{tabular}

MIC: Minimum inhibitory concentration

If the dose of arbekacin is formulated as 4 times the normal dose with dose spacing of $72 \mathrm{~h}$, it will have the following benefits: (i) The dose will cross 4 times the normal MIC which is well above minimum bactericidal concentration (MBC) and for that the chance of crossresistance or resistant mutant will be nil. (ii) If the dose is 4 times increased, it will be far less than the toxic dose and therefore safe. The renal-related adverse drug reactions of arbekacin are increased with a higher Ctrough [11]. The incidence of arbekacin induced nephrotoxicity was observed when it was administrated at a total dose of over $5000 \mathrm{mg}$ [20]. Moreover, with a dose spacing of $72 \mathrm{~h}$, the Ctrough will be lower. (iii) Outpatient antimicrobial therapy is possible in resource-limited settings. (iv) It can be cost effective and can be used in remote places where a basic sensitivity pattern is available.

Hence, in case of severe sepsis, we can plan the treatment in the following way - first, send the culture from all relevant sites and give arbekacin $800 \mathrm{mg}$ (4 times the normal dose to attend the MBC). No antibiotics are needed for the next $72 \mathrm{~h}$ until the culture sensitivity report comes and plan the antibiotics accordingly. All other supportive measures are to be continued as per the sepsis protocol. The total cost of therapy will be less with a fair chance of patient survival.

\section{CONCLUSION}

Hence, in this drug-resistant era, arbekacin can be used as an empirical choice in severe sepsis as monotherapy or in combination with other antibiotics such as colistin or polymyxin to fight against MDR and XDR bugs. The favorable pharmacokinetics, pharmacodynamics, and spectrum (simultaneous Gram-positive and Gram-negative coverage) of the drug with the advantage of a single daily dose will make the antibiotic as a handy choice for the management of sepsis with least time to the thermometer to needle even in resource-limited health-care settings in India.

\section{AUTHORS' CONTRIBUTIONS}

Dr. Soma Sarkar - Data collection and manuscript writing, Dr. Dipankar Sarkar - Design the research study, Dr. Anjum Namhata - Preparation of manuscript, and Dr. Manideepa Sengupta - Editing of the manuscript as per journal guidelines.

\section{CONFLICTS OF INTEREST}

The authors declare that they have no conflicts of interest.

\section{AUTHORS' FUNDING}

The study was self-funded.

\section{REFERENCES}

1. Dixit A, Kumar N, Kumar S, Trigun V. Antimicrobial resistance: Progress in the decade since emergence of New Delhi metallo- $\beta$ lactamase in India. Indian J Community Med 2019;44:4-8.

2. Shamsuzzaman SM. Multidrug-resistant, extensively drug-resistant and pandrug-resistant Bacteria and antimicrobial therapy in combination. Bangladesh J Med Microbiol 2015;9:1-2.

3. Savoia D. New antimicrobial approaches: Reuse of old drugs. Curr Drug Targets 2016;17:731-8.

4. Tanaka N, Matsunaga K, Hirata A, Matsuhisa Y, Nishimura T. Mechanism of action of habekacin, a novel amino acid containing aminoglycoside antibiotic. Antimicrob Agents Chemother 1983;24:797-802.

5. Panchal G, Pandit R, Trailokya A, Sharma A. Arbekacin-a novel antibiotic for critical infections. J Assoc Physicians India 2019;67:93-7.

6. Kondo S. Development of arbekacin and synthesis of new derivatives stable to enzymatic modifications by methicillin-resistant Staphylococcus aureus. Jpn J Antibiot 1994;47:561-74.

7. Hotta K, Kondo S. Kanamycin and its derivative, arbekacin: Significance and impact. J Antibiot 2018;71:417-24.

8. Matsumoto T. Arbekacin: Another novel agent for treating infections due to methicillin-resistant Staphylococcus aureus and multidrugresistant gram-negative pathogens. Clin Pharmacol 2014;6:139-48.

9. Matsuhashi $\mathrm{Y}$, Yamamoto $\mathrm{H}$. The enzymatic mechanisms of resistance to aminoglycoside antibiotics in methicillin-cephem-resistant Staphylococcus aureus. Jpn J Antibiot 1988;41:523-9.

10. Aoki Y. Bactericidal activity of arbekacin against methicillin-resistant Staphylococcus aureus. Comparison with that of vancomycin. Jpn J Antibiot 1994;47:640-6.

11. Sato R, Tanigawara Y, Kaku M, Aikawa N, Shimizu K. Pharmacokineticpharmacodynamic relationship of arbekacin for treatment of patients infected with methicillin-resistant Staphylococcus aureus. Antimicrob Agents Chemother 2006;50:3763-9.

12. Watanabe T, Ohashi K, Matsui K, Kubota T. Comparative studies of the bactericidal, morphological and post-antibiotic effects of arbekacin and vancomycin against methicillin-resistant Staphylococcus aureus. J Antimicrob Chemother 1997;39:471-6.

13. Lee J, Kim CK, Roh KH, Lee H, Yum JH, Yong D, et al. In vitro activity of arbekacin against clinical isolates of Staphylococcus species and gram-negative bacilli. Korean J Lab Med 2007;27:292-7.

14. Zapor MJ, Barber M, Summers A, Miller GH, Feeney LA, Eberly LE, et al. In vitro activity of the aminoglycoside antibiotic arbekacin against Acinetobacter baumannii calcoaceticus isolated from war-wounded patients at Walter reed army medical center. Antimicrob Agents Chemother 2010;54:3015-7.

15. Araoka H, Baba M, Tateda K, Ishii Y, Oguri T, Okuzumi K, et al. In vitro combination effects of aztreonam and aminoglycoside against multidrug-resistant Pseudomonas aeruginosa in Japan. Jpn J Infect Dis 2012;65:84-7.

16. Watanabe A, Yanagihara K, Matsumoto T, Kohno S, Aoki N, Oguri T, et al. Nationwide surveillance of bacterial respiratory pathogens conducted by the surveillance committee of Japanese society of chemotherapy, Japanese association for infectious diseases, and Japanese society for clinical microbiology in 2009: General view of the pathogens' 
antibacterial susceptibility. J Infect Chemother 2012;18:609-20.

17. Kazuno Y, Tsuneta S, Tamra A, Shiraishi S, Takada H, Kasai T, et al. Bactriological evaluation of a new aminoglycoside antibiotic, HBK. Chemotherapy 1986;34:61-71.

18. Funatsu Y, Hasegawa N, Fujiwara H, Namkoong H, Asami T, Tasaka S, et al. Pharmacokinetics of arbekacin in bronchial epithelial lining fluid of healthy volunteers. J Infect Chemother 2014;20:607-11.

19. Okada K, Kimura T, Mikamo H, Kasahara K, Seki M, Takakura S, et al. Clinical practice guidelines for therapeutic drug monitoring of arbekacin: A consensus review of the Japanese society of chemotherapy and the Japanese society of therapeutic drug monitoring. J Infect Chemother 2014;20:1-5.

20. Negita K, Yamashita M, Kubota T, Sugiura Y, Miura T, Katsumi A, et al. Study on therapeutic drug monitoring of arbekacin in patients infected with methicillin-resistant Staphylococcus aureus and its efficacy. Jpn J Pharm Health Care Sci 2001;27:123-31. 